\title{
Prevalence of infectious multi-drug resistant bacteria isolated from immunocompromised patients in Tunisia
}

\author{
Arij Mechergui, Wafa Achour, Sondos Mathlouthi, Assia Ben Hassen
}

The National Bone Marrow Transplant Center, 1006, Tunis, Tunisia.

Faculty of Medicine of Tunis, UR12ES02, 1007, Tunis, Tunisia.

\begin{abstract}
Objectives: A retrospective study was conducted in the Bone Marrow Transplant Center of Tunisia during a period of 10 years (from 2002 to 2011) in order to report the prevalence of infectious multi-drug resistant bacteria.

Methods: Bacterial identification was carried on the basis of biochemical characteristics and API identification systems. Antibiotic susceptibility was tested by disc diffusion method on Muller-Hinton agar.

Results: During the study period, 34.5\% of 142 Klebsiella pneumoniae strains and $11.46 \%$ of 218 Escherichia coli strains were extended-spectrum beta-lactamase (ESBL) producers. Also, 32.8\% of 210 strains of Psendomonas aeruginosa were imipenem and/or ceftazidime resistant and $20.75 \%$ of 106 strains of Staphylococcus aureus were methicillin resistant. A rising trend was observed for the prevalence of the selected multidrug resistant bacteria.

Conclusion: These findings may have important clinical implications in prophylaxis and selection of antibiotic treatment. Continuous surveillance is needed, especially for onco-hematological patients.

Keywords: Infectious multi-drug resistant bacteria, immunocompromised patients, Tunisia.

DOI: https://dx.doi.org/10.4314/ahs.v19i2.25

Cite as: Mechergui A, Achour W, Mathlouthi S, Hassen AB. Prevalence of infectious multi-drug resistant bacteria isolated from immunocompromised patients in Tunisia. Afri Health Sci.2019;19(2): 2021-2025. https:/ / dx.doi.org/10.4314/abs.v19i2.25
\end{abstract}

\section{Introduction}

Increasing antibiotic resistance in bacteria is a cause of concern in the treatment of infections, particularly in hematopoietic stem cell transplant patients who have a greater propensity toward acquiring infections because of the underlying immunosuppression. Multidrug-resistant infections pose a major quandary for clinicians by complicating therapy choice, compromising patient recovery, and creating a serious threat to public health ${ }^{1}$.

Multidrug resistant (MDR) organisms are defined as microorganisms that are resistant to one or more classes of antimicrobial agents ${ }^{2}$. In Northern Africa, there is a

\section{Corresponding author:}

Arij Mechergui,

Laboratory unit, the National Bone Marrow

Transplant Center, Djebel-Lakdhar Street,

Bab Saadoun, 1006, Tunis, Tunisia

Phone: +21671565623

Fax: +21671565623

E-mail: arij.mechergui@gmail.com paucity of data concerning MDR profiles in hematology centers .

Organisms such as methicillin-resistant Staphylococcus aureus, imipenem and/or ceftazidime resistant Pseudomonas aeruginosa, and ESBL producing Enterobacteriaceae have become problematic at variable frequencies in different transplantation centers. These organisms can be acquired through the gastrointestinal tract early after hematopoietic stem cell transplantation and later through multiple different routes, especially in people in whom endogenous flora have been altered due to prolonged or recurrent antibiotic exposure. In addition, bacteria have the ability of easily transfer genes, which contributes to perpetuation of the resistant species ${ }^{3}$.

Facing the growing problem of bacterial resistance, the aim of this study was to evaluate the MDR prevalence among hematological cell transplant patients, to provide information to fight against these organisms spread.

\section{Materials and methods}

Bacterial collection

From 2002 to 2011, infectious bacteria isolated from pa- 
tients monitored in the Bone Marrow Transplant Center of Tunisia, were screened for multidrug resistance. Only one representative isolate from each specimen per patient, regardless of clinical significant isolates, was included in the analysis. Bacterial isolates included in the present study were ESBL producing Escherichia coli and Klebsiella pneumoniae, imipenem and/or ceftazidime resistant $P$. aeruginosa and methicillin resistant $S$. aureus.

\section{Patients}

Allogeneic stem cells recipients were hospitalized in laminar air-flow rooms, whereas autologous stem cells recipients were treated in single conventional rooms. All patients received non absorbable oral antibiotic (colimycin and gentamycin). Oral amphotericin B was administered as antifungal prophylaxis. Antibacterial prophylaxis with fluoroquinolones was not given. No systemic antibiotic was used as routine prophylaxis. The initial empirical treatment of neutropenic fever consisted of piperacilline-tazobactam associated with amikacin or ciprofloxacin. Glycopeptides or intravenous amphotericin B deoxycholate are used in second or third line therapy.

\section{Bacterial identification}

Bacterial identification was carried on the basis of standard cultural, morphological and biochemical characteristics (Gram staining, catalase and oxydase tests) and by the API identification systems (bioMérieux, Marcy-l'Étoile, France).

\section{Antimicrobial susceptibility testing}

Antibiotic susceptibility of the isolates was tested by disc diffusion method according to the recommendations of the Antibiogram Committee of the French Society for Microbiology (http://www.sfm-microbiologie.org/). E. coli CIP 7624 (ATCC 25922) was used as reference strain for antibiotic susceptibility testing quality control. Also, external quality controls were conducted regularly by the Tunisian health ministry.

\section{Ethics statement}

This study was performed with approval from the Local Medical Ethical Committee of Charles Nicolle Hospital, Tunis, Tunisia. As the strains were deidentified and analyzed anonymously, and the strains, not a human, were studied, this is exempt from human research committee approval according to the regulations of the Local Medical Ethical Committee of Charles Nicolle Hospital, Tunis, Tunisia and informed consent is not required according to the Ethical Committee.

\section{Results}

In the study period, we isolated 218 E. coli, 210 P. aeruginosa, 142 K. pneumoniae and 106 S. aureus (Table1).

The rate of ESBL producing strains was of $34.5 \%$ (49/142) for K. pneumoniae and $11.46 \%$ for E. coli (25/218). Imipenem and/or ceftazidime resistance rate accounted for 32.8\% (69/210) in P. aeruginosa. Methicillin resistant S. aureus was of $20.75 \%(22 / 106)$ (Table 1).

Table 1. Distribution of MDR bacteria rates by species

\begin{tabular}{|c|c|c|c|c|c|}
\hline & P. aeruginosa & K. pneumoniae & E. coli & S. aureus & Total \\
\hline Total isolates & 210 & 142 & 218 & 106 & 676 \\
\hline MDR \% & $69(32.8 \%)$ & $49(34.5 \%)$ & $25(11.46 \%)$ & $22(20.75 \%)$ & $165(24.4 \%)$ \\
\hline $\begin{array}{l}\text { MDR rates } / 1000 \\
\text { patients-days }\end{array}$ & 1.32 & 0.94 & 0.48 & 0.42 & 3.03 \\
\hline
\end{tabular}


The prevalence of MRSA increased from 0 to 0.22 per 1,000 patient days. The frequency of ESBL producing organisms trended up from 0 to 1.32 per 1,000 patient days for K. pneumoniae and from 0.25 to 1.55 per 1,000 patient days for E. coli. The rate of imipenem and/or ceftazidime resistance increased from 2.07 to 2.21 per 1,000 patient days for $P$. aeruginosa (Figure 1 ).

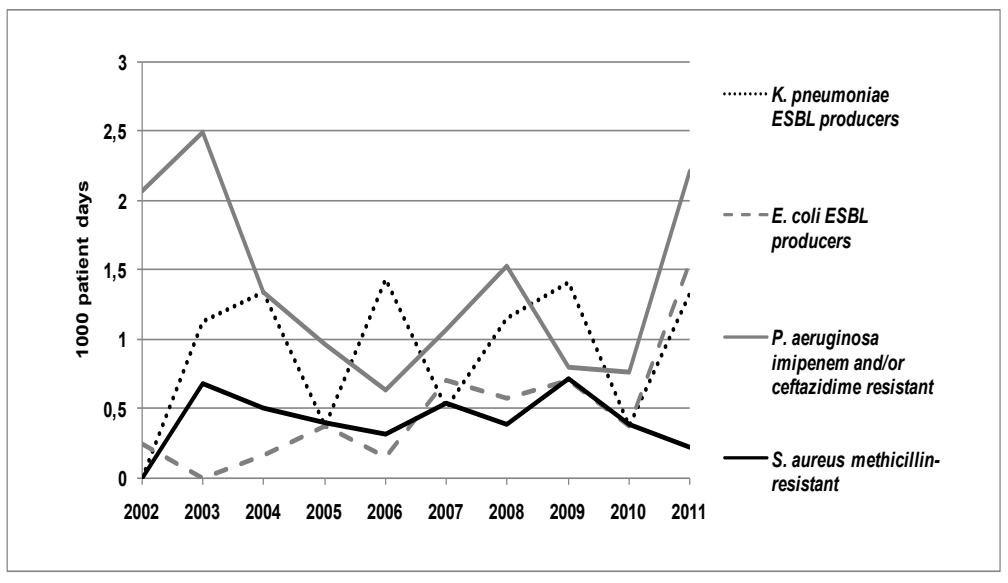

Figure 1. Percentage of MDR bacteria rates expressed by 1,000 patient days

\section{Discussion}

Infections caused by MDR organisms are associated with increased mortality, hospital length of stay, and health care costs ${ }^{4}$. Patients with cancer are exposed to a wide range of infections. Many of the challenges surrounding infection control are the same for patients with cancer as for other hospital in-patients ${ }^{5}$.

In our study, the most common MDR organisms were $K$. pneumoniae $(34.5 \%)$ and $P$. aeruginosa $(32.8 \%)$ followed by S. aureus $(20.75 \%)$ and E. coli $(11.46 \%)$. These differences in MDR organisms incidence may be the evidence that measures taken for the global MDR organisms reduction may have distinct effect for each micro-organism.

Methicillin-resistant $S$. aureus is the most important cause of antibiotic-resistant healthcare-associated infections worldwide. Also, MRSA bloodstream infections can cause significant morbidity and mortality in patients with cancer ${ }^{6}$. In the study period, an increasing trend was observed among our MRSA isolates from 0 to 0.22 per 1,000 patient days. Similarly, the rate of MRSA trended up from 0.3 to 1.0 isolates/1000 patient in an American hematological malignancy and transplantation unit, from 1999 to 20047. In contrast, the rate of MRSA health care-associated infection declined 3.4 fold in an American liver transplant intensive care unit from 4.1 per 1,000 patient days during $2001-2003$ to 1.2 per 1,000 patient days during 2004-2006 ${ }^{8}$.
In our study, $20.75 \%$ of our $S$. aureus strains were methicillin resistant. Similarly, 23\% of Pennsylvanian patients who received liver transplants develop MRSA infections, from 1990 through $1998^{9}$. The same percentage of MRSA $(23 \%)$ is found in an American study conducted from 1999 to 2006, among S. aureus causing bacteremia after allogeneic hematopoietic stem cell transplantation ${ }^{10}$. MRSA account for 36\% in German patients with a bone marrow or peripheral blood, from 2000 to $2003^{11}$. MRSA rate among American patients with febrile neutropenia, from 1999 to 2004, is approximately four times higher than that reported in our study ( $80 \%$ vs $20.75 \%)$.

ESBL-producing $K$. pneumoniae may cause serious infections such as bacteremia, pneumonia, and urinary tract infection especially in critically ill patients ${ }^{12}$. In our study, the frequency of $K$. pneumoniae ESBL producers organisms trended up from 0 to 1.32 per 1,000 patient days. In an American neonatal intensive care unit, the incidence of $K$. pneumoniae ESBL infection peaked from 0 in June 2000 to 4.1 cases per 1,000 patient-days in April 2001 ${ }^{13}$. During this study period, the level of $K$. pneumoniae ESBL producers reached $34.5 \%$. This rate was two times lower than that reported in a Korean blood and marrow transplantation center, from 2009 to 2010, among K. pneumoniae ESBL producers causing blood stream infections $(34.5 \%$. vs $71.0 \%)$. A comparable rate $(37.8 \%)$ was found among $K$. pneumoniae ESBL-producers strains causing 
bloodstream infection in a Brazilian cancer center, from 2000 to $2002^{15}$. A higher rate of $51.6 \%$ is found in ESBL K. pneumoniae bloodstream infection among Malaysian febrile neutropenic patients, between 1996 and $1997^{16}$.

E. coli is the most common Gram-negative bacterium causing bacteremia among neutropenic hosts ${ }^{17}$. The frequency of our E. coli ESBL producers strains increased from 0.25 to 1.55 per 1,000 patient days, in the study period. In contrast, ESBL-producing E. coli had non-significant decrease from 0.38 to 0.11 per 100 patient-days in a Brazilian non-teaching hospital ${ }^{18}$. In our center, E. coli ESBL producing was of $11.46 \%$. A comparable rate of $12.6 \%$ is found in E. coli ESBL strains causing bacteraemia among Spanish patients with cancer ${ }^{19}$. According to a Brazilian study conducted in a cancer center from 2000 to 2002, E. coli ESBL producing strains isolated from bloodstream infection were of $8.9 \%{ }^{15}$. In contrast, ESBL producers accounted for $31.9 \%$ of E. coli strains of bloodstream infections in a Korean blood and marrow transplantation center, during the period from 2009 to $2010^{14}$. A much higher rate of E. coli ESBL producers $(55 \%)$ is found among Texan patients with hematologic malignancies, during the period from 2003 to $2007^{20}$. The rate of acute prostatitis caused by E. coli ESBL after transrectal prostate biopsy was of $43 \%$ in a Turkish study conducted from 2003 to $2008^{21}$.

MDR $P$. aeruginosa strains are increasing in frequency ${ }^{22}$ and have been very recently described as a growing problem also in adult onco-hematologic patients ${ }^{23}$. In our study, the imipenem and/or ceftazidime resistance rate among $P$. aeruginosa strains increased from 2.07 to 2.21 per 1,000 patient days. In a Brazilian non-teaching hospital, the rate of imipenem resistant- $P$. aeruginosa decreased from 1.37 per 100 patient-days (June-December 2002) to 0.78 per 100 patient-days (December 2002-May 2003) ${ }^{18}$. $32.8 \%$ of our $P$. aeruginosa strains were imipenem and/ or ceftazidime resistant. In a Chinese hematology and oncology department, resistance rates are found to be $0 \%$ for imipenem and 100\% for ceftazidime among $P$. aeruginosa strains isolated from patients with bloodstream infections, between January and December $2010^{24}$. In an Italian study, imipenem and ceftazidime resistance rates are respectively determined to $24 \%$ and $30 \%$ among $P$. aeruginosa isolated from infection in children undergoing chemotherapy and hematopoietic stem cell transplantation, from 2000 to $2008^{25}$. According to a Brazilian study, imipenem and ceftazidime resistance rates are respective- ly found as $80 \%$ and $100 \%$ among $P$. aeruginosa isolated from bacteremia among hematopoietic stem cell transplant recipients, in $2004^{26}$. An Italian hematology ward report an imipenem and ceftazidime resistance rates of $74 \%$ and $31 \%$ respectively, among $P$. aeruginosa strains during a study conducted from 1998 to $1999^{27}$.

\section{Conclusion}

During this study period, we reported a significant increase in the incidence of the selected MDR bacteria. Thus, systematic screening of multi-drug resistant bacteria carriage is needed to be continued in our center especially because of the high frequency of ESBL producing $K$. pneumoniae and E. coli in our country. A multidisciplinary approach is needed, involving oncologists, microbiologists, and infection-control personnel.

\section{Conflict of interest}

We declare that there is no conflict of interest regarding the publication of this article.

\section{References}

1. Boucher HW, Talbot GH, Bradley JS et al. Bad bugs, no drugs: no eskape! An update from the infectious diseases society of america. Clin Infect Dis. 2009; 48:1-12.

2. Siegal DJ, Rhinehart E, Jackson M, Chiarello L : Multidrug-Resistant organisms in Healthcare Settings. Healthcare Infection Control Practices Advisory Committee. HICPAC; 2006:1-74.

3. Rossolini GM, Mantengoli E, Montagnani F, et al. Epidemiology and clinical relevance of microbial resistance determinants versus anti-Gram-positive agents. Curr Opin Microbiol, 2010; 13: 582-8.

4. Cosgrove S. The relationship between antimicrobial resistance and patient outcomes: mortality, length of hospital stay, and healthcare costs. Clin Infect Dis. 2006;42:S82-S89.

5. Kamboj M, Sepkowitz KA. Nosocomial infections in patients with cancer. Lancet oncol. 2009;10:589-97.

6. Mahajan SN, Shah JN, Hachem R et al. Characteristics and outcomes of methicillin-resistant Staphylococcus aureus bloodstream infections in patients with cancer treated with vancomycin: 9-year experience at a comprehensive cancer center. The Oncologist. $2012 ; 17$ :1329-1336.

7. Craig M, Cumpston AD, Hobbs GR et al. The clinical impact of antibacterial prophylaxis and cycling antibiotics for febrile neutropenia in a hematological malignancy and 
transplantation unit. Bone Marrow Transplantation. 2007; 398: 477-482.

8. Russell DL, Flood A, Zaroda TE et al. Outcomes of colonization with MRSA and VRE among liver transplant candidates and recipients. American Journal of Transplantation. 2008; 8: 1737-1743.

9. Singh N, Paterson DL, Chang FY et al. Methicillin-resistant Staphylococcus aureus: the other emerging resistant gram-positive coccus among liver transplant recipients. Clinical Infectious Diseases. 2000; 30, 322-327.

10. Mihu CN, Schaub J, Kesh S et al. Risk factors for late Staphylococcus aureus bacteremia after allogeneic hematopoietic stem cell transplantation: A Single-Institution, Nested Case-Controlled Study. Biol Blood Marrow Transplant. 2008;14:1429-33.

11. Dettenkofer M, Wenzler-Röttele S, Babikir R et al. Surveillance of nosocomial sepsis and pneumonia in patients with a bone marrow or peripheral blood stem cell transplant: a multicenter project. Clinical infectious diseases. 2005; 40: 926-931.

12. Laurent $C$, Rodriguez-Villalobos $H$, Rost F et al. Intensive care unit outbreak of extended-spectrum $\beta$-Lactamase-producing Klebsiella pneumoniae controlled by cohorting patients and reinforcing infection control measures. Infection Control and Hospital Epidemiology. 2008; 296: 517-524.

13. Gupta A, Della-Latta P, Todd B et al. Outbreak of extended-spectrum beta-lactamase-producing Klebsiella pneumoniae in a neonatal intensive care unit linked to artificial nails. Infection Control and Hospital Epidemiology. 2004; 25: 210-215.

14. Kwon JC, Kim SH, Choi JK et al. Epidemiology and clinical features of bloodstream infections in hematology wards: one year experience at the Catholic Blood and Marrow Transplantation Center. Infection \& Chemotherapy. 2013; 45: 51-61.

15. Velasco E, Byington R, Martins CSA et al. Bloodstream infection surveillance in a cancer centre: a prospective look at clinical microbiology aspects. Clinical Microbiology and Infection. 2004; 10: 542-549.

16. Ariffin H, Navaratnam P, Mohamed M et al. Ceftazidime-resistant Klebsiella pneumoniae bloodstream infection in children with febrile neutropenia. International Journal of Infectious Diseases. 2000; 4: 21-25.

17. Sepkowitz KA. Antibiotic prophylaxis in patients re- ceiving hematopoietic stem cell transplant. Bone Marrow Transplantation. $2002 ; 29$ : 367-371

18. Furtado GHC, Perdiz LB, Medeiros EAS. The Effect of a 4th Generation-Cephalosporin Introduction upon the Incidence of Multidrug-Resistant Gram-Negative Bacteria in a Non-Teaching Hospital. American Journal of Infectious Diseases. 2008; 44, 267.

19. Gudiol C, Calatayud L, Garcia-Vidal C et al. Bacteraemia due to extended-spectrum beta-lactamase-producing Escherichia coli ESBL-EC in cancer patients: clinical features, risk factors, molecular epidemiology and outcome. Journal of Antimicrobial Chemotherapy. 2010; 65:333-41.

20. Vigil KJ, Johnson JR, Johnston BD et al. Escherichia coli pyomyositis: an emerging infectious disease among patients with hematologic malignancies. Clinical infectious diseases. 2010; 50: 374-380.

21. Özden E, Bostanci Y, Yakupoglu KY et al. Incidence of Acute Prostatitis Caused by Extended-spectrum $\beta$-Lactamase-producing Escherichia coli After Transrectal Prostate Biopsy. Urology. 2009;741: 119-123.

22. Giske CG, Monnet DL, Cars O et al. Clinical and economic impact of common multidrug-resistant gram-negative bacilli. Antimicrobial Agents and Chemotherapy. 2008;523:813-21

23. Rangaraj G, Granwehr BP, Jiang Y et al. Perils of quinolone exposure in cancer patients: breakthrough bacteremia with multidrug-resistant organisms. Cancer. 2010;1164:967-73

24. Lv H, Ning B. Pathogenesis of bloodstream infection in children with blood cancer. Experimental and therapeutic medicine. 2013; 5: 201-204.

25. Caselli D, Cesaro S, Ziino O et al. Multidrug resistant Pseudomonas aeruginosa infection in children undergoing chemotherapy and hematopoietic stem cell transplantation. Haematologica. 2010; 959: 1612-1615.

26. Oliveira AL, de Souza M, Carvalho-Dias VMH et al. Epidemiology of bacteremia and factors associated with multi-drug-resistant gram-negative bacteremia in hematopoietic stem cell transplant recipients. Bone Marrow Transplantation. 2007; 39: 775-781

27. Lang A, De FG, Meyer R et al. Comparison of antimicrobial use and resistance of bacterial isolates in a haematology ward and an intensive care unit. European Journal of Clinical Microbiology and Infectious Diseases. 2001; 20: 657-660. 